Canad. Math. Bull. Vol. 23 (4), 1980

\title{
SPHERICAL HARMONICS ON THE HEISENBERG GROUP
}

\author{
BY \\ PETER C. GREINER*
}

1. The Heisenberg group, $H_{1}$. Equip $\mathbb{R}^{3}$ with the group law

$$
(z, t)\left(z^{\prime}, t^{\prime}\right)=\left(z+z^{\prime}, t+t^{\prime}+2 \operatorname{Im} z \bar{z}^{\prime}\right),
$$

where $(z, t)$ stands for $(x, y, t)$. This is a nilpotent Lie group, usually referred to as the first Heisenberg group, $H_{1}$. In general $H_{k}$ denotes $\mathbb{R}^{2 k+1}$ equipped with a similar group law, namely

$$
(z, t)\left(z^{\prime}, t^{\prime}\right)=\left(z+z^{\prime}, t+t^{\prime}+2 \operatorname{Im} z \cdot \bar{z}^{\prime}\right),
$$

where $z=\left(z_{1}, \ldots, z_{k}\right), z_{j}=x_{j}+i y_{j}, j=1, \ldots, k$ and $z \cdot \bar{z}^{\prime}=\sum_{l=1}^{k} z_{l} \bar{z}_{l}^{\prime}$. In this article we work only on $H_{1}$, although everything we say carries over to $H_{k}$. A basis for the left-invariant vector fields on $H_{1}$ is given by $Z, \bar{Z}$ and $T$, where

$$
\begin{gathered}
Z=\frac{\partial}{\partial z}+i \bar{z} \frac{\partial}{\partial t}, \\
T=\frac{\partial}{\partial t}
\end{gathered}
$$

and, as usual

$$
\frac{\partial}{\partial z}=\frac{1}{2}\left(\frac{\partial}{\partial x}-i \frac{\partial}{\partial y}\right)
$$

A real basis is given by $X, Y$ and $T$, where $Z=(X-i Y) / 2$ i.e.

$$
X=\frac{\partial}{\partial x}+2 y \frac{\partial}{\partial t}, \quad Y=\frac{\partial}{\partial y}-2 x \frac{\partial}{\partial t} .
$$

Let $\mathscr{L}_{0}$ denote the following second order left-invariant differential operator on $H_{1}$ :

$$
\mathscr{L}_{0}=-\frac{1}{2}(Z \bar{Z}+\bar{Z} Z)=-\frac{\partial^{2}}{\partial z \partial \bar{z}}+i \frac{\partial}{\partial t}\left(z \frac{\partial}{\partial z}-\bar{z} \frac{\partial}{\partial \bar{z}}\right)-|z|^{2} \frac{\partial^{2}}{\partial t^{2}} .
$$

Received by the editors, October 10, 1979.

* This research was partially supported by the Natural Sciences and Engineering Research Council of Canada under Grant A-3017.

This paper is one of a series of survey papers written at the invitation of the Editors of the Bulletin. 
$\mathscr{L}_{0}$ is not elliptic. Nevertheless, Folland showed in [2], that

$$
\mathscr{L}_{0} \Phi_{0}=\delta,
$$

where

$$
\Phi_{0}=\frac{1}{2 \pi}\left(|z|^{4}+t^{2}\right)^{-1 / 2},
$$

and $\delta$ stands for the delta function at the origin. Translating $\Phi_{0}$ on $H_{1}$ yields the fundamental solution of $\mathscr{L}_{0}$ on $\mathbb{R}^{3}$. This suggests that, in analogy with the theory of the Laplacian on $\mathbb{R}^{3},\left(|z|^{4}+t^{2}\right)^{1 / 4}$ may be thought of as the distance of $(z, t)$ from the origin, and via left translation one obtains a distance function on $H_{1}$.

Given $R>0$, one introduces the dilation $R:(z, t) \rightarrow\left(R z, R^{2} t\right)$. Then $\mathscr{L}_{0}$ is a homogeneous differential operator of degree two, in the sense that

$$
\mathscr{L}_{0} f\left(R z, R^{2} t\right)=R^{2}\left(\mathscr{L}_{0} f\right)\left(R z, R^{2} t\right) .
$$

Continuing in this vein there is a great deal of similarity between the behaviour of $\mathscr{L}_{0}$ on $H_{1}$ and that of the Laplacian on $\mathbb{R}^{3}$. This is the theme of this article, namely, to exploit this analogy in introducing solid spherical harmonics for operators like $\mathscr{L}_{0}$ on $H_{1}$.

2. Parallels between $H_{1}$ and $\mathbb{R}^{3}$. I shall begin by constructing two tables, one for $\mathbb{R}^{3}$ and one for $H_{1}$, of analogous properties and results.

$$
\mathbb{R}^{3}:
$$

(2.1 $\left.1_{\mathrm{E}}\right)$ Euclidean translation, i.e., vector addition.

$\left(2.2_{\mathrm{E}}\right)$ Euclidean dilation:

$$
\begin{aligned}
R:\left(x_{1}, x_{2}, x_{3}\right) & \rightarrow\left(R x_{1}, R x_{2}, R x_{3}\right), \quad R>0 . \\
\Delta & =\frac{\partial^{2}}{\partial x_{1}^{2}}+\frac{\partial^{2}}{\partial x_{2}^{2}}+\frac{\partial^{2}}{\partial x_{3}^{2}},
\end{aligned}
$$

the Laplacian, is a second order, (left and right) invariant differential operator on $\mathbb{R}^{3}$, homogeneous of degree two with respect to Euclidean dilation, i.e. $\Delta f(R x)=R^{2}(\Delta f)(R x)$.

$\left(2.4_{\mathrm{E}}\right) r=r(x)=\left(x_{1}^{2}+x_{2}^{2}+x_{3}^{2}\right)^{1 / 2}$ is the distance of $x=\left(x_{1}, x_{2}, x_{3}\right)$ from the origin. Then $r\left(x-x^{\prime}\right)$ is the Euclidean distance of $x$ and $x^{\prime}$. 1.

$\left(2.5_{\mathrm{E}}\right)$ The Euclidean unit ball, $B_{\mathrm{E}}(1)$, is defined by $r(x)=\left(x_{1}^{2}+x_{2}^{2}+x_{3}^{2}\right)^{1 / 2}<$

(2.6 $\left.6_{\mathrm{E}}\right)$ The Dirichlet problem is solvable for $\Delta$ on $B_{\mathrm{E}}(1)$ :

Let $\phi \epsilon C\left[b B_{\mathrm{E}}(1)\right]$, i.e., $\phi$ is a continuous function on the boundary of $B_{\mathrm{E}}(1)$. 
Then there is a unique continuous function $u$ on the closure of $B_{\mathrm{E}}(1)$, so that $u\left[b B_{\mathrm{E}}(1)\right]=\phi$, and $\Delta u=0$ in $B_{\mathrm{E}}(1)$ in the sense of distributions. is.

$\left(2.7_{E}\right) \Delta$ is real-analytic hypoelliptic, i.e. $\Delta u=f \Rightarrow u$ is real-analytic whenever $f$

$$
H_{1} \text { : }
$$

$\left(2.1_{\mathrm{H}}\right)$ The Heisenberg group law (1.1) replaces Euclidean translation.

$\left(2.2_{\mathrm{H}}\right)$ The Heisenberg dilation is given by

$$
R:(z, t) \rightarrow\left(R z, R^{2} t\right), \quad R>0 .
$$

$\left(2.3_{\mathrm{H}}\right) \mathscr{L}_{0}=-\frac{1}{2}(Z \bar{Z}+\bar{Z} Z)$-see (1.5)-is a second order differential operator on $\mathbb{R}^{3}$, left-invariant on $H_{1}$, homogeneous of degree two with respect to the Heisenberg dilation, i.e. $\mathscr{L}_{0} f\left(R z, R^{2} t\right)=R^{2}\left(\mathscr{L}_{0} f\right)\left(R z, R^{2} t\right), R>0$.

$\left(2.4_{H}\right) \rho=\rho(z, t)=\left(|z|^{4}+t^{2}\right)^{1 / 4}$ denotes the Heisenberg distance of $(z, t)$ from the origin. Then $\left(\left|z-z^{\prime}\right|^{4}+\left(t-t^{\prime}-2 \operatorname{Im} z \bar{z}^{\prime}\right)^{2}\right)^{1 / 4}$ is the Heisenberg distance of $(z, t)$ from $\left(z^{\prime}, t^{\prime}\right)$.

$\left(2.5_{\mathrm{H}}\right)$ The Heisenberg unit ball is defined by $\rho=\left(|z|^{4}+t^{2}\right)^{1 / 4}<1$. In the French literature this is often referred to as "Boule de Korànyi"

(2.6 $\left.6_{\mathrm{H}}\right)$ The Dirichlet problem is solvable for $\mathscr{L}_{0}$ on $B_{\mathrm{H}}(1)$ :

Let $\phi \in b B_{\mathrm{H}}(1)$. Then there is a unique continuous function $u$ on the closure of $B_{\mathrm{H}}(1)$, so that $u\left(b B_{\mathrm{H}}(1)\right)=\phi$, and $\mathscr{L}_{0} u=0$ in $B_{\mathrm{H}}(1)$ in the sense of distributions.

$\left(2.7_{\mathrm{H}}\right) \mathscr{L}_{0}$ is real-analytic hypoelliptic, i.e., $\mathscr{L}_{0} u=f \Rightarrow u$ is real-analytic whenever $f$ is.

A few remarks are in order. Properties (2.1), (2.2), (2.3), (2.4) and (2.5) are formal structures. (2.6) and (2.7) are deep results. In particular (2.6 $)$ and $\left(2.7_{\mathrm{E}}\right)$ are classical. A proof of $\left(2.6_{\mathrm{H}}\right)$ can be found in [4] and Folland's fundamental solution-see [2] - gives $\left(2.7_{\mathrm{H}}\right)$.

3. Spherical harmonics: heuristics. Properties (2.1)-(2.7) suggest the study of spherical harmonics on the respective unit balls. Here we shall sketch an argument which justifies this statement. Consider $\Delta$ first.

Let $\phi \in C\left[b B_{\mathrm{E}}(1)\right]$. By $\left(2.6_{\mathrm{E}}\right)$ there is a unique function $u$, continuous on the closure of $B_{\mathrm{E}}(1)$, such that $u\left(b B_{\mathrm{E}}(1)\right)=\phi$, and $\Delta u=0$ in $B_{\mathrm{E}}(1)$ in the sense of distributions.

According to $\left(2.7_{\mathrm{E}}\right) u$ is real-analytic in $B_{\mathrm{E}}(1)$. In particular, $u$ can be represented as uniformly and absolutely convergent power series in some neighbourhood of the origin. Applying $\Delta$ to the power series term-by-term, (2.3 $3_{\mathrm{E}}$ implies that homogeneous parts of the power series must be in the 
null-space of $\Delta$. Thus in some neighbourhood of the origin $u$ can be written as a (usually infinite) sum of homogeneous harmonic polynomials. This argument suggests that it is of some importance to study homogeneous harmonic polynomials, i.e., the solid spherical harmonics and their boundary values, the spherical harmonics. In particular this leads to the representation of harmonic functions in terms of their boundary value via the Poisson kernel.

This argument carries over, word for word, to $\mathscr{L}_{0}$ as long as homogeneity is understood in the Heisenberg sense-see $\left(2.2_{\mathrm{H}}\right)$-i.e., $z, \bar{z}$ are $H$ homogeneous of degree one and $t$ is $H$-homogeneous of degree two. Thus we see that $\mathscr{L}_{0}$-harmonic functions have a representation near the origin as a (usually infinite) sum of $H$-homogeneous polynomials which are $\mathscr{L}_{0}$-harmonic.

(3.1) Definition. A solid $\mathscr{L}_{0}$-spherical harmonic of degree $m, m=0,1$, $2, \ldots$ is a polynomial in $z, \bar{z}$ and $t$, which is harmonic with respect to $\mathscr{L}_{0}$ and which is homogeneous of degree $m$ with respect to the Heisenberg dilation, $\left(2.2_{\mathbf{H}}\right)$.

The purpose of this article is to compute explicitly the $H$-homogeneous $\mathscr{L}_{0}$-harmonic polynomials, i.e., the solid $\mathscr{L}_{0}$-spherical harmonics and their boundary value on $b B_{\mathrm{H}}(1)$, the $\mathscr{L}_{0}$-spherical harmonics.

4. Spherical coordinates on $H_{1}$. Imitating $\mathbb{R}^{3}$ we set

$$
\begin{gathered}
x=\rho \sin ^{1 / 2} \phi \cos \theta, \quad y=\rho \sin ^{1 / 2} \phi \sin \theta, \\
t=\rho^{2} \cos \phi,
\end{gathered}
$$

with $0<\rho<\infty, 0 \leq \phi \leq \pi$ and $0 \leq \theta<2 \pi$. In other words

$$
\begin{gathered}
\rho=\left(|z|^{4}+t^{2}\right)^{1 / 4}, \\
z=|z| e^{i \theta}=\rho \sin ^{1 / 2} \phi e^{i \theta}, \\
t+i|z|^{2}=\rho^{2} e^{i \phi} .
\end{gathered}
$$

A bit of calculation yields

$$
\begin{gathered}
Z=\frac{\partial}{\partial z}+i \bar{z} \frac{\partial}{\partial t} \\
=e^{-i \theta}\left(\frac{1}{2} i \sin ^{1 / 2} \phi e^{-i \phi} \frac{\partial}{\partial \rho}+\frac{\sin ^{1 / 2} \phi}{\rho} e^{-i \phi} \frac{\partial}{\partial \phi}-\frac{1}{2} i \frac{1}{\rho \sin ^{1 / 2} \phi} \frac{\partial}{\partial \theta}\right) .
\end{gathered}
$$

We shall also need

$$
\frac{\partial}{\partial \mathrm{t}}=\frac{\cos \phi}{2 \rho} \frac{\partial}{\partial \rho}-\frac{\sin \phi}{\rho^{2}} \frac{\partial}{\partial \phi} .
$$

5. The differential operators $\mathscr{L}_{\alpha}$. In [3] Folland and Stein introduced the 
operators $\mathscr{L}_{\alpha}, \alpha \in \mathbb{C}$, given by

$$
\begin{aligned}
\mathscr{L}_{a} & =-\frac{1}{2}(Z \bar{Z}+\bar{Z} Z)+i \alpha \frac{\partial}{\partial t} \\
& =\mathscr{L}_{0}-\frac{1}{2} \alpha[Z, \bar{Z}],
\end{aligned}
$$

where $[Z, \bar{Z}]=Z \bar{Z}-\bar{Z} Z$. These operators behave like $\mathscr{L}_{0}$. Thus $\mathscr{L}_{\alpha}$ is leftinvariant on $H_{1}$ and homogeneous of degree two, i.e.

$$
\mathscr{L}_{\alpha}\left(f\left(R z, R^{2} t\right)\right)=R^{2}\left(\mathscr{L}_{\alpha} f\right)\left(R z, R^{2} t\right), \quad R>0 .
$$

This follows easily, e.g., from (4.6) and (4.7). Furthermore $\mathscr{L}_{\alpha}$ is hypoelliptic, i.e., $\mathscr{L}_{\alpha} u=f \in C^{\infty} \Rightarrow u \in C^{\infty}$, and solvable if and only if $\alpha \neq \pm 1, \pm 3, \pm 5, \ldots$ When $\pm \alpha=1,3,5, \ldots \mathscr{L}_{\alpha}$ has a fundamental solution given by $\Phi_{\alpha}\left(z-z^{\prime}, t-\right.$ $\left.t^{\prime}-2 \operatorname{Im} z \bar{z}^{\prime}\right)$, i.e.

$$
\mathscr{L}_{\alpha} \Phi_{\alpha}\left(z-z^{\prime}, t-t^{\prime}-2 \operatorname{Im} z \bar{z}^{\prime}\right)=\delta_{\left(z^{\prime}, t^{\prime}\right)},
$$

where $\delta_{\left(z^{\prime}, t^{\prime}\right)}$ is the delta function of $\left(z^{\prime}, t^{\prime}\right)$ and where

$$
\Phi_{\alpha}(z, t)=c_{\alpha}\left(|z|^{4}+t^{2}\right)^{-1 / 2}\left(\frac{|z|^{2}+i t}{|z|^{2}-i t}\right)^{\alpha / 2}
$$

with

$$
c_{\alpha}=\frac{1}{\pi^{2}} \Gamma\left(\frac{1+\alpha}{2}\right) \Gamma\left(\frac{1-\alpha}{2}\right)
$$

When $\alpha= \pm 1, \pm 3, \pm 5, \ldots$ one obtains so-called relative fundamental solutions-see [5], [6] and [7].

6. The spherical harmonics for $\mathscr{L}_{\alpha}, \pm \alpha \neq 1,3,5, \ldots$ Instead of studying $\mathscr{L}_{0}$ we shall compute the spherical harmonics for $\mathscr{L}_{\alpha}$. Since the interest in spherical harmonics is mainly to understand Dirichlet's problem on the unit ball from now on we shall assume that $\mathscr{L}_{\alpha}$ is solvable, i.e. $\pm \alpha \neq 1,3,5, \ldots$ In trying to find homogeneous harmonic polynomials for $\mathscr{L}_{\alpha}$ the first problem one encounters is that $\mathscr{L}_{\alpha}$ does not separate in $(\rho, \theta, \phi)$ coordinates. To get an idea of what these solid spherical harmonics for $\mathscr{L}_{\alpha}$ look like I computed some explicitly for $\mathscr{L}_{0}$. This turned out to be useful. Here are some of the examples.

Let $\mathscr{H}_{m}^{(0)}$ denote the set of homogeneous harmonic polynomials of degree $m$ for $\mathscr{L}_{0}$. Then

$\mathscr{H}_{0}^{(0)}=\{1\}$,

$\mathscr{H}_{1}^{(0)}=\{z, \bar{z}\}$,

$\mathscr{H}_{2}^{(0)}=\left\{z^{2}, \bar{z}^{2}, t\right\}$

$\mathscr{H}_{3}^{(0)}=\left\{z^{3}, \bar{z}^{3}, z\left(|z|^{2}-2 i t\right), \bar{z}\left(|z|^{2}+2 i t\right)\right\}$,

$\mathscr{H}_{4}^{(0)}=\left\{z^{4}, \bar{z}^{4}, z^{2}\left(|z|^{2}-\frac{3}{2} i t\right), \bar{z}^{2}\left(|z|^{2}+\frac{3}{2} i t\right),|z|^{4}-2 t^{2}\right\}, \ldots$ etc. 
Here $\{\alpha, \beta, \ldots\}$ denotes the linear span of $\alpha, \beta, \ldots$. Introducing spherical coordinates we obtain

$$
\begin{aligned}
\mathscr{H}_{0}^{(0)}= & \{1\}, \\
\mathscr{H}_{1}^{(0)}= & \left\{e^{i \theta} \rho \sin ^{1 / 2} \phi, e^{-i \theta} \rho \sin ^{1 / 2} \phi\right\}, \\
\mathscr{H}_{2}^{(0)}= & \left\{e^{2 i \theta} \rho^{2} \sin \phi, e^{-2 i \theta} \rho^{2} \sin \phi, \rho^{2} \cos \phi\right\}, \\
\mathscr{H}_{3}^{(0)}= & \left\{e^{3 i \theta} \rho^{3} \sin ^{3 / 2} \phi, e^{-3 i \theta} \rho^{3} \sin ^{3 / 2} \phi, e^{i \theta} \rho^{3} \sin ^{3 / 2} \phi\left(1-2 i \frac{t}{|z|^{2}}\right),\right. \\
& \left.e^{-i \theta} \rho^{3} \sin ^{3 / 2} \phi\left(1+2 i \frac{t}{|z|^{2}}\right)\right\}, \\
\mathscr{H}_{4}^{(0)}= & \left\{e^{4 i \theta} \rho^{4} \sin ^{2} \phi, e^{-4 i \theta} \rho^{4} \sin ^{2} \phi, e^{2 i \theta} \rho^{4} \sin ^{2} \phi\left(1-\frac{3}{2} i \frac{t}{|z|^{2}}\right),\right. \\
& \left.e^{-2 i \theta} \rho^{4} \sin ^{2} \phi\left(1+\frac{3}{2} i \frac{t}{|z|^{2}}\right), \rho^{4} \sin ^{2} \phi\left(1-2\left(\frac{t}{|z|^{2}}\right)^{2}\right)\right\}, \ldots \text { etc. }
\end{aligned}
$$

From these formulas we can immediately make a guess at the form of the general solid spherical harmonic of $\mathscr{L}_{0}$, and, also, of that of $\mathscr{L}_{\alpha}$. It turns out to be correct. First a definition.

(6.1) Definition. A solid $\mathscr{L}_{\boldsymbol{\alpha}}$-spherical harmonic of degree $m, m=$ $0,1,2, \ldots$ is a polynomial in $z, \bar{z}$ and $t$ which is harmonic with respect to $\mathscr{L}_{\alpha}$ and which is homogeneous with respect to the Heisenberg dilation, $\left(2.2_{\mathbf{H}}\right)$, i.e. $H$-homogenous.

(6.2) Proposition. All solid spherical harmonics of $\mathscr{L}_{\alpha}$ of degree $m, m=$ $0,1,2, \ldots$ are sums of $H$-homogeneous $\mathscr{L}_{\alpha}$-harmonic polynomials of degree $m$ of the form

$$
e^{i n \theta} \rho^{m} \sin ^{m / 2} \phi h(\cot \phi),
$$

where $|n| \leq m, m \equiv n(\bmod 2)$ and $h(x)$ is some polynomial in $x$-which, of course depends on $\alpha, n$ and $m$.

Proof: We note that

$$
\mathscr{L}_{\alpha}\left(e^{i n \theta} f(|z|, t)\right)=e^{i n \theta} \mathscr{L}_{\alpha, n} f(|z|, t),
$$

where $\mathscr{L}_{\alpha, n}$ is a second order differential operator in $r=|z|$ and $t$. Let $u(z, t)$ be a $H$-homogeneous $\mathscr{L}_{\alpha}$-harmonic polynomial of degree $m$. We may write it, uniquely, as follows.

$$
u(z, t)=\sum_{n} e^{i n \theta} u_{n}(|z|, t)
$$

Then

$$
\mathscr{L}_{\alpha}(u)=\sum_{n} e^{i n \theta} \mathscr{L}_{\alpha, n} u_{n}(|z|, t)=0
$$


which implies that $\mathscr{L}_{\alpha, n} u_{n}(|z|, t)=0$ for all $n$. Therefore, from (6.4) we have

$$
\mathscr{L}_{\alpha}\left(e^{i n \theta} u_{n}(|z|, t)\right)=0
$$

for all $n$. The general term of $u$ is some constant times

$$
z^{m_{1}} \bar{z}^{m_{2}} t^{m_{3}}=e^{i\left(m_{1}-m_{2}\right) \theta}|z|^{m_{1}+m_{2}} t^{m_{3}}=e^{i n \theta}|z|^{m}\left(\frac{t}{|z|^{2}}\right)^{m_{3}},
$$

where $m_{1}+m_{2}+2 m_{3}=m$ and $n=m_{1}-m_{2}$. In particular $m \equiv n(\bmod 2),|n| \leq$ $m$, and since $|z|^{m}=\rho^{m} \sin ^{m / 2} \phi$ and $t /|z|^{2}=\cot \phi$, we have derived Proposition 6.2 .

7. Calculating $h(x)=h_{(m-|n|) / 2}^{(\alpha, n)}(x)$. This requires the explicit computation of

$$
\mathscr{L}_{\alpha}(u)=-Z \bar{Z} u+i(\alpha-1) \frac{\partial u}{\partial t},
$$

where

$$
u=e^{i n \theta} \rho^{m} \sin ^{m / 2} \phi y(\cot \phi) .
$$

With a certain amount of work we obtain

$$
\begin{aligned}
\mathscr{L}_{\alpha}(u)= & -e^{i n \theta} \rho^{m} \sin ^{m / 2} \phi\left\{\left(1+\cot ^{2} \phi\right) y^{\prime \prime}(\cot \phi)\right. \\
& -[(m-1) \cot \phi+i(\alpha+n)] y^{\prime}(\cot \phi) \\
& \left.+\frac{1}{4}\left(m^{2}-n^{2}\right) y(\cot \phi)\right\} .
\end{aligned}
$$

We assume $\mathscr{L}_{\alpha}(u)=0$ and then look for a polynomial solution, $y(x)$, of

$$
\left(1+x^{2}\right) y^{\prime \prime}(x)-(i(\alpha+n)+(m-1) x) y^{\prime}(x)+\frac{1}{4}\left(m^{2}-n^{2}\right) y(x)=0 .
$$

To find the required solution we substitute

$$
y=\sum_{\nu=0}^{\infty} a_{\nu}(x-i)^{\nu}
$$

in (7.4). This leads to

$$
\begin{gathered}
(x+i) \sum_{\nu=2}^{\infty} \nu(\nu-1) a_{\nu}(x-i)^{\nu-1}-(i(\alpha+n)+(m-1) x) \sum_{\nu=1}^{\infty} \nu a_{\nu}(x-i)^{\nu-1} \\
+\frac{m^{2}-n^{2}}{4} \sum_{\nu=0}^{\infty} a_{\nu}(x-i)^{\nu}=0
\end{gathered}
$$

which yields the recurrence relation

$$
i(2 \nu-\alpha-n-m+1)(\nu+1) a_{\nu+1}+\left(\nu^{2}-m \nu+\frac{m^{2}-n^{2}}{4}\right) a_{\nu}=0
$$

for $\nu=0,1,2,3, \ldots$. Now $m+n$ is even and by hypothesis $\pm \alpha \neq 1,3,5$, $7, \ldots$. Therefore the coefficient of $a_{\nu+1}$ in (7.7) never vanishes. Thus $y(x)$ is a polynomial if and only if the coefficient of $a_{\nu}$ in (7.7) vanishes at some point, 
i.e.

$$
0=\nu^{2}-m \nu+\frac{m^{2}-n^{2}}{4}=\left(\nu-\frac{m-|n|}{2}\right)\left(\nu-\frac{m+|n|}{2}\right)
$$

Since $|n| \leq m$, (7.4) has a polynomial solution, which is unique up to a constant multiplier, of degree

$$
k=\frac{1}{2}(m-|n|)
$$

exactly. We set $a_{0}=1$ in (7.5). Then

$$
a_{\nu}=\left(\frac{i}{2}\right)^{\nu} \frac{\left(-\frac{m-|n|}{2}\right)_{\nu}\left(-\frac{m+|n|}{2}\right)_{\nu}}{\left(-\frac{m+n}{2}-\frac{\alpha-1}{2}\right)_{\nu}} \frac{1}{\nu !},
$$

$\nu=1,2,3, \ldots$ where we used the standard notation

$$
(a)_{0}=1,(a)_{n}=a(a+1) \cdots(a+n-1), \quad n=1,2,3, \ldots .
$$

Thus the polynomial solution of (7.4) is given by

$$
\begin{aligned}
& y(x)=\sum_{\nu=0}^{s / 2(m-|n|)}\left(\frac{i}{2}\right)^{\nu} \frac{\left(-\frac{m-|n|}{2}\right)_{\nu}\left(-\frac{m+|n|}{2}\right)_{\nu}}{\left(-\frac{m+n}{2}-\frac{\alpha-1}{2}\right)_{\nu}} \frac{(x-i)^{\nu}}{\nu !} \\
& =F\left(-\frac{m-|n|}{2},-\frac{m+|n|}{2} ;-\frac{m+n}{2}-\frac{\alpha-1}{2} ; \frac{1}{2}+\frac{1}{2} i x\right) .
\end{aligned}
$$

Here $F(a, b ; c ; z)$ stands for Gauss' hypergeometric function defined by

$$
F(a, b ; c ; z)=\sum_{\nu=0}^{\infty} \frac{(a)_{\nu}(b)_{\nu}}{(c)_{\nu}} \frac{z^{\nu}}{\nu !}
$$

as long as $c \neq 0,-1,-2,-3, \ldots$. We note that in our case

$$
c=-\frac{m+n}{2}-\frac{\alpha-1}{2} \neq 0,-1,-2,-3, \ldots
$$

since $\pm \alpha \neq 1,3,5, \ldots$. For sufficiently small $z$

$$
F(a, b ; c ; z)=(1-z)^{-a} F\left(a, c-b ; c ; \frac{z}{z-1}\right),
$$

as long as $c \neq 0,-1,-2,-3, \ldots$-ee [1], v. 1, p. 105(3). If $a=0,-1,-2$, $-3, \ldots$ both sides of (7.14) are polynomials, hence (7.14) holds for all $z \in \mathbb{C}$. In our case $a=-k=-(m-|n|) / 2$ is a nonpositive integer, therefore, disregarding constant multipliers, (7.12) can be written in the following form

$$
y(x)=(x+i)^{(m-|n|) / 2} F\left(-\frac{m-|n|}{2}, \frac{|n|-n}{2}-\frac{\alpha-1}{2} ;-\frac{m+n}{2}-\frac{\alpha-1}{2} ; \frac{x-i}{x+i}\right) .
$$


This formula can be further simplified in the following way. With $k$ a non-negative integer we have

$$
\begin{aligned}
& \left(\begin{array}{c}
k+b \\
k
\end{array}\right)(x+i)^{k} F\left(-k,-k-a ; b+1 ; \frac{x-i}{x+i}\right) \\
& =\left(\begin{array}{c}
k+b \\
k
\end{array}\right)(x+i)^{k} \sum_{\nu=0}^{k} \frac{k(k-1) \cdots(k-\nu+1)}{(b+1)(b+2) \cdots(b+\nu)}\left(\begin{array}{c}
k+a \\
\nu
\end{array}\right)\left(\frac{x-i}{x+i}\right)^{\nu} \\
& =(x+i)^{k} \sum_{\nu=0}^{k} \frac{(k+b)(k+b-1) \cdots(b+\nu+1)}{1 \cdot 2 \cdots(k-\nu)}\left(\begin{array}{c}
k+a \\
\nu
\end{array}\right)\left(\frac{x-i}{x+i}\right)^{\nu} \\
& =\sum_{\nu=0}^{k}\left(\begin{array}{c}
k+a \\
\nu
\end{array}\right)\left(\begin{array}{c}
k+b \\
k-\nu
\end{array}\right)(x-i)^{\nu}(x+i)^{k-\nu} .
\end{aligned}
$$

Thus, with $k=(m-|n|) / 2, \quad k+a=-(|n|-n) / 2+(\alpha-1) / 2 \quad$ and $\quad b=$ $-(m+n) / 2-(\alpha+1) / 2$ we derived

(7.17) Proposition. $h(x)=h_{(m-|n|) / 2}^{(\alpha, n)}(x)$, with $(m-|n|) / 2=0,1,2, \ldots$, is the unique, up to a constant multiplier, polynomial solution of (7.4). $h_{k}^{(\alpha, n)}(x), k=0$, $1,2, \ldots$ is exactly of degree $k$. Explicitly, one has

$$
h_{k}^{(\alpha, n)}(x)=\sum_{\nu=0}^{k}\left(\begin{array}{c}
-\frac{|n|+n}{2}-\frac{\alpha+1}{2} \\
\nu
\end{array}\right)\left(\begin{array}{c}
-\frac{|n|-n}{2}+\frac{\alpha-1}{2} \\
k-\nu
\end{array}\right)(x+i)^{\nu}(x-i)^{k-\nu}
$$

8. The $\mathscr{L}_{\alpha}$-spherical harmonics: final version. Using Propositions 6.2 and 7.17 the basis elements of the solid $\mathscr{L}_{\alpha}$-spherical harmonics of degree $m$ have the form

$$
e^{i n \theta} \rho^{m} \sin ^{m / 2} \phi h_{(m-|n|) / 2}^{(\alpha, n)}(\cot \phi),
$$

where $n=0, \pm 1, \pm 2, \pm 3, \ldots$ such that $(m-|n|) / 2=0,1,2,3, \ldots$. We shall simplify this. With $x=\cot \phi$

$$
x+i=\frac{\cos \phi}{\sin \phi}+i=\frac{e^{i \phi}}{\sin \phi}, x-i=\frac{e^{-i \phi}}{\sin \phi} .
$$

Therefore

(8.2) $\sin ^{k} \phi h_{k}^{(\alpha, n)}(\cot \phi)$

$$
\begin{aligned}
& =\sum_{\nu=0}^{k}\left(\begin{array}{c}
-\frac{|n|+n}{2}-\frac{\alpha+1}{2} \\
\nu
\end{array}\right)\left(\begin{array}{c}
-\frac{|n|-n}{2}+\frac{\alpha-1}{2} \\
k-\nu
\end{array}\right) \cdot e^{i \nu \phi} e^{-i(k-\nu) \phi} \\
& =\sum_{\nu=0}^{k}\left(\begin{array}{c}
-\frac{|n|+n}{2}-\frac{\alpha+1}{2} \\
\nu
\end{array}\right)\left(\begin{array}{c}
-\frac{|n|-n}{2}+\frac{\alpha-1}{2} \\
k-\nu
\end{array}\right) \cdot e^{i(2 \nu-k) \phi} .
\end{aligned}
$$


We set

$$
H_{k}^{(\alpha, n)}\left(e^{i \phi}\right)=(-1)^{k} \sum_{\nu=0}^{k}\left(\begin{array}{c}
-\frac{|n|+n}{2}-\frac{\alpha+1}{2} \\
\nu
\end{array}\right)\left(\begin{array}{c}
-\frac{|n|-n}{2}+\frac{\alpha-1}{2} \\
k-\nu
\end{array}\right) e^{i(2 \nu-k)}
$$

Then

$$
\sin ^{k} \phi h_{k}^{(\alpha, n)}(\cot \phi)=(-1)^{k} H_{k}^{(\alpha, n)}\left(e^{i \phi}\right) .
$$

Now we are ready to state our main result. Collecting the formulas we obtained so far, we have derived

(8.5) Theorem. Set

$$
\mathscr{L}_{\alpha}=-Z \bar{Z}+i(\alpha-1) \frac{\partial}{\partial t}, \quad \pm \alpha \neq 1,3,5, \ldots
$$

Then the linear space $\mathscr{H}_{m}^{(\alpha)}$ of $H$-homogeneous $\mathscr{L}_{\alpha}$-harmonic polynomials of degree $m, m=0,1,2, \ldots$ has dimension $m+1$. A basis for $\mathscr{H}_{m}^{(\alpha)}$ can be found as follows:

For each $n$ and $k$, such that $m=2 k+|n|$ with $k=0,1,2,3, \ldots$ and $n=0, \pm 1$, $\pm 2, \pm 3, \ldots$ set

$$
\mathscr{H}_{m}^{(\alpha, n)}(\rho, \theta, \phi)=e^{i n \phi} \rho^{m} \sin ^{|n| / 2} \phi H_{(m-|n|) / 2}^{(\alpha, n)}\left(e^{i \phi}\right),
$$

where $H_{k}^{(\alpha, n)}\left(e^{i \phi}\right)$ is defined by the generating function

$$
\begin{gathered}
\left(1-\rho e^{i \phi}\right)^{-(|n|+n) / 2-(\alpha+1) / 2}\left(1-\rho e^{-i \phi}\right)^{-(|n|-n) / 2+(\alpha-1) / 2} \\
=\sum_{k=0}^{\infty} \rho^{k} H_{k}^{(\alpha, n)}\left(e^{i \phi}\right),
\end{gathered}
$$

with the right hand side converging for $\rho<1$.

Then $\left\{\mathscr{H}_{m}^{(\alpha, n)}\right.$, all possible $\left.n\right\}$ is a basis for $\mathscr{H}_{m}^{(\alpha)}$.

(8.8) Definition. The $\mathscr{L}_{\alpha}$-spherical harmonics are obtained by restricting $H$-homogeneous $\mathscr{L}_{\alpha}$-harmonic polynomials to $b B_{H}(1)$, i.e. to $\rho=1$.

(8.9) THEOREM. Each $\mathscr{L}_{\alpha}$-spherical harmonic is a unique linear combination of functions of the form

$$
e^{i n \theta} \sin ^{|n| / 2} \phi H_{k}^{(\alpha, n)}\left(e^{i \phi}\right)
$$

with $k=0,1,2,3, \ldots$ and $n=0, \pm 1, \pm 2, \pm 3, \ldots$

9. Solid spherical harmonics of $\Delta$. It is interesting to recall the homogeneous harmonic polynomials of degree $m, m=0,1,2, \ldots$ of the Laplacian $\Delta$ in $\mathbb{R}^{3}$. In spherical coordinates they are

$$
e^{i n \theta} r^{m} \sin ^{|n|} \phi P_{m-|n|}^{|n|+1 / 2}(\cos \phi),
$$


$0 \leq \theta<2 \pi, 0 \leq \phi \leq \pi$ and $0<r<\infty$, where $n=0, \pm 1, \pm 2, \ldots, \pm m$ and the ultraspherical (or Gegenbauer) polynomials, $P_{k}^{\lambda}(x),-1 \leq x \leq 1$, are defined via the generating function

$$
\left(1-r e^{i \phi}\right)^{-\lambda}\left(1-r e^{-i \phi}\right)^{-\lambda}=\left(1-2 r \cos \phi+r^{2}\right)^{-\lambda}=\sum_{k=0}^{\infty} r^{k} P_{k}^{\lambda}(\cos \phi),
$$

see [10], section 4.7. $P_{k}^{\lambda}$ are sometimes denoted by $C_{k}^{\lambda}-$ see e.g. [1], v. 2, p. $240(22,23)$ and p. 177 (29). The central result in the theory of the polynomials $P_{k}^{\lambda}(x)$ is

(9.3) Proposition. For each $\lambda>-\frac{1}{2}$ the polynomials $P_{k}^{\lambda}(x), k=0,1,2,3, \ldots$ form an orthogonal basis for $L^{2}([-1,1])$ with measure $\left(1-x^{2}\right)^{\lambda-1 / 2} d x$. Furthermore

$$
\int_{-1}^{1}\left(P_{k}^{\lambda}(x)\right)^{2}\left(1-x^{2}\right)^{\lambda-1 / 2} d x=\frac{\pi^{1 / 2}(2 \lambda)_{k} \Gamma\left(\lambda+\frac{1}{2}\right)}{(k+\lambda) k ! \Gamma(\lambda)}, \quad k=0,1,2, \ldots
$$

For a proof of Proposition 9.3, sections 10.3 and 10.9 of [1], v. 2, may be consulted. It follows easily from (9.2) that with $l=0,1,2,3, \ldots$

$$
P_{k}^{l+1 / 2}(\cos \phi)=H_{k}^{(-2 l,-2 l)}\left(e^{i \phi}\right), \quad k=0,1,2, \ldots .
$$

In particular, ‘when $l=0$ we obtain the Legendre polynomials

$$
P_{k}(\cos \phi)=P_{k}^{1 / 2}(\cos \phi)=H_{k}^{(0,0)}\left(e^{i \phi}\right), \quad k=0,1,2, \ldots
$$

10. On the convergence of solid spherical harmonic expansions. We shall exhibit a $\mathscr{L}_{0}$-harmonic function on the whole open Heisenberg unit ball, $B_{H}(1)$, whose expansion in solid spherical harmonics near the origin does not converge on all of $B_{H}(1)$. This is in contrast to the classical spherical harmonic expansions of the Laplacian in the Euclidean unit ball.

We choose $2 \pi \Phi_{0}(z-1, t-2 \operatorname{Im} z)$ for our harmonic function in $B_{H}(1)$, where $\Phi_{0}(z-1, t-2 \operatorname{Im} z)$ is the fundamental solution of $\mathscr{L}_{\mathrm{o}}$ with the singularity at $\left(z^{\prime}, t^{\prime}\right)=(1,0)$. Thus $\Phi_{0}$ is real analytic in all of $B_{H}(1)$, in particular it is real analytic on $b B_{H}(\rho)$ for all $\rho<1$, and has an expansion in solid $\mathscr{L}_{0}$-spherical harmonics which converges near the origin. This can be written as

$$
\begin{aligned}
2 \pi \Phi_{0}(z-1, t-2 \operatorname{Im} z)= & \left(|z-1|^{4}+(t-2 \operatorname{Im} z)^{2}\right)^{-1 / 2} \\
= & \left(i \rho^{2} e^{-i \phi}-2 \rho \sin ^{1 / 2} \phi e^{i \theta}+1\right)^{-1 / 2} \\
& \cdot\left(-i \rho^{2} e^{i \phi}-2 \rho \sin ^{1 / 2} \phi e^{-i \theta}+1\right)^{-1 / 2} \\
= & \sum_{k=0}^{\infty} \rho^{k} v_{k}(\phi, \theta),
\end{aligned}
$$

where $\rho^{k} v_{k}(\phi, \theta)$ are the solid $\mathscr{L}_{\mathrm{o}}$-spherical harmonics of degree $k$. The right hand side of (10.1) converges for all $\phi$ and $\theta$ if $\rho$ is sufficiently small. The explicit form of $v_{k}(\phi, \theta)$ is irrelevant for this discussion. Fix a direction, $(\phi, \theta)$, 
in (10.1) and let $\rho$ take on complex values. Then the radius of convergence of the power series in $\rho$, in (10.1), is the minimum of the lengths of the complex $\rho$-solutions of

$$
i \rho^{2} e^{-i \phi}-2 \rho \sin ^{1 / 2} \phi e^{i \theta}+1=0 .
$$

In particular, choosing $\phi=\theta=\pi / 2$, the solutions of

$$
\rho^{2}-2 i \rho+1=0
$$

are $i(1 \pm \sqrt{ } 2)$ and the minimum length of these is $\sqrt{ } 2-1<\frac{1}{2}$, which proves our contention. Thus we have derived

(10.4) Proposition. The solid $\mathscr{L}_{0}$-spherical harmonic expansion of $2 \pi \Phi_{0}(z-$ $1, t-2 \operatorname{Im} z)$ converges in some $B_{H}(\rho), \rho>0$. The largest such $\rho$ is less than $\frac{1}{2}$.

Of course, the first part of Proposition 10.4 follows from $\left(2.7_{\mathbf{H}}\right)$.

As we mentioned earlier this is in contrast to the classical case of the Laplacian in $\mathbb{R}^{3}$, whose harmonic functions in the Euclidean unit ball, $B_{\mathrm{E}}(1)$, have a solid spherical harmonic expansion which converges in all of $B_{\mathrm{E}}(1)$. To be more precise, we shall give a quick sketch of the relevant result following Müller's treatment of this subject in [9].

Let $\Omega_{3}$ denote $b B_{E}(1)$ in $\mathbb{R}^{3}$ and $\omega_{3}$ its surface area. If $F(\xi)$ is a continuous function on $\Omega_{3}$ then the Poisson integral

$$
u(r, \xi)=\frac{1}{\omega_{3}} \int_{\Omega_{3}} \frac{\left(1-r^{2}\right) F(\eta)}{\left(1-2 r \xi \cdot \eta+r^{2}\right)^{3 / 2}} d \omega_{3}(\eta)
$$

represents the unique harmonic function in $B_{E}(1)$ which is continuous on the closure of $B_{\mathrm{E}}(1)$, and whose restriction to $\Omega_{3}=b B_{\mathrm{E}}(1)$ is $F(\xi)$. Now

$$
\frac{1-r^{2}}{\left(1-2 r \xi \cdot \eta+r^{2}\right)^{3 / 2}}=\sum_{k=0}^{\infty} N_{k} r^{k} P_{k}(\xi \cdot \eta),
$$

where

$$
\sum_{k=0}^{\infty} N_{k} r^{k}=\frac{1+r}{(1-r)^{2}}
$$

and $P_{k}(x)$ are the Legendre polynomials-see (9.6). Since $|P(x)| \leq 1$ on $-1 \leq$ $x \leq 1$, (10.7) implies that for all $r<1$ (10.6) converges, uniformly in $\xi, \eta \in \Omega_{3}$. Integrating on $\Omega_{3}$ we obtain

(10.8) Proposition. Let $F(\xi)$ be a continuous function on $\Omega_{3}$. Then

$$
u(r, \xi)=\sum_{k=0}^{\infty} r^{k} S_{k}(\xi)
$$


with

$$
S_{k}(\xi)=\frac{N_{k}}{\omega_{3}} \int_{\Omega_{3}} P_{k}(\xi \cdot \eta) F(\eta) d \omega_{3}(\eta),
$$

converges for all $r<1$, uniformly in $\xi \in \Omega_{3}$, and represents the harmonic function in $B_{\mathrm{E}}(1)$ with $F(\xi)$ for its boundary value. $r^{k} S_{k}(\xi)$ are the solid spherical harmonics of degree $k$.

Finally, to use the analogy with Proposition 10.4, we compute the roots of the denominator of the Poisson kernel in (10.5). Thus

$$
r^{2}-2 r(\xi \cdot \eta)+1=0 \Rightarrow r_{1,2}=e^{ \pm i \xi \cdot \eta}
$$

and both roots have unit length. This should be compared to the behaviour of solutions of (10.3).

Remark. Proposition 10.4 suggests the possibility that the $\mathscr{L}_{\alpha}$-spherical harmonics, or, more specifically $H_{k}^{(\alpha, n)}\left(e^{i \phi}\right), k=0,1,2,3, \ldots$ may not form an orthogonal system.

A more elaborate study of $\Phi_{0}(z-w, t-s-2 \operatorname{Im} z \bar{w})$ may yield an answer to the following question.

Question. What is the largest $\rho$, call it $\rho_{\max }$, with the property, that all functions which are $\mathscr{L}_{0}$-harmonic in $B_{\mathrm{H}}(1)$ and continuous on the closure of $B_{\mathrm{H}}(1)$ have a convergent solid $\mathscr{L}_{\mathrm{o}}$-spherical harmonic expansion in $B_{\mathrm{H}}\left(\rho_{\text {max }}\right)$ ?'

11. Conclusion. An interesting question is whether the functions $H_{k}^{(\alpha, n)}$ $\left(e^{i \phi}\right), k=0,1,2, \ldots$, with $n=0, \pm 1, \pm 2, \ldots$ fixed, are complete on $0 \leq \phi \leq \pi$-e.g. the solvability of the Dirichlet problem for $\mathscr{L}_{\theta}$ on $B_{\mathrm{H}}(1)$ is strong evidence for the probable completeness of $H_{k}^{(0, n)}, k=0,1,2, \ldots$. Such a result would allow one to construct the Poisson kernel for the Dirichlet problem for $\mathscr{L}_{\alpha}, \alpha \neq \pm 1, \pm 3, \pm 5, \ldots$ on $B_{\mathrm{H}}(1)$. It may also yield information about the $\bar{\partial}_{b}$-Neumann problem for $\mathscr{L}_{n-2}$ in the unit Heisenberg ball in $H_{n}$, $n>1-$ see [8]. At this moment this is idle speculation, which may, however, lead to interesting results in the future.

\section{REFERENCES}

1. A. Erdélyi, et al., Higher Transcendental functions, v. 1 and v. 2, Bateman Manuscript Project, McGraw-Hill, New York, 1955.

2. G. B. Folland, A fundamental solution for a subelliptic operator, Bull. Amer. Math. Soc. 79 (1973), pp. 373-376.

3. G. B. Folland and E. M. Stein, Estimates for the $\bar{\partial}_{b}$-complex and analysis on the Heisenberg group, Comm. Pure Appl. Math. 27 (1974), pp. 429-522.

4. B. Gaveau, Principe de moindre action, propagation de la chaleur et estimees sous elliptiques sur certains groupes nilpotents, Acta Math. 139 (1977), pp. 95-153.

5. P. C. Greiner, J. J. Kohn and E. M. Stein, Necessary and sufficient conditions for the solvability of the Lewy equation, Proc. Nat. Acad. of Sciences, U.S.A., 72 (1975), pp. 3287-3289. 
6. P. C. Greiner and E. M. Stein, Estimates for the $\bar{\partial}-$ Neumann problem, Mathematical Notes Series, no. 19, Princeton Univ. Press, Princeton, N.J., 1977.

7. - On the solvability of some differential operators of type $\square_{b}$, Proc. of the Seminar on Several Complex Variables, Cortona, Italy, 1976-1977, pp. 106-165.

8. M. Kuranishi, A priori estimate in strongly pseudo-convex CR structure over small balls, Preprint.

9. C. Müller, Spherical harmonics, Lecture Notes in Math., v. 17, Springer-Verlag, Berlin, 1966.

10. G. Szegö, Orthogonal polynomials, Amer. Math. Soc. Colloquium Publications, v. 23, Amer. Math. Soc., Providence, R.I., 1939.

\section{DEPARTMENT OF MATHEMATICS \\ UNIVERSTTY OF TORONTO \\ TORONTO, CANADA \\ M5S 1A1}

